\title{
ARTICLE
}

\section{Thermal neutron field with D-T neutron source for BNCT}

\author{
Makoto SAKAI*, Naohiro FUJIMOTO, Katsunori ISHII, \\ Isao MURATA and Kunio AWAZU
}

Graduate School of Engineering, Osaka University, 2-1, Yamadaoka, Suita, Osaka, Japan

\begin{abstract}
BNCT (Boron Neutron Capture Therapy) is one of the less invasive cancer treatments. BNCT is effective especially for brain cancer, however a little inefficient at present because a nuclear reactor is necessary as a neutron source. If high boron concentration would be realized, accelerator-based BNCT could be realized, because the dose of BNCT is determined by the product of the ${ }^{10} \mathrm{~B}$ concentration and neutron fluence. The intracellular boron concentration using Hemagglutinating Virus of Japan envelope (HVJ-E) would become more than 100 times compared with conventional boron compound (Sodium borocaptate: BSH). In this study, we constructed a thermal neutron field for BNCT with a D-T neutron source at the intense $14 \mathrm{MeV}$ neutron source facility OKTAVIAN of Osaka University.

The thermal neutron flux distribution in the irradiation assembly was calculated by MCNP and compared with experimental results. From the result, we confirmed an acceptable thermal neutron field could be constructed for basic irradiation experiments for HVJ-E, and the flux intensity would be $\sim 2.2 \times 10^{-4} \mathrm{n} / \mathrm{cm}^{2} /$ source neutron. From the result of in-vitro experiments, an anti-tumor effect was observed. The series study showed the potential of the accelerator-based BNCT using the novel boron agent, HVJ-E.
\end{abstract}

KEYWORDS: BNCT, accelerator, HVJ-E, BSH, tumor, D-T neutron

\section{Introduction}

Recently the number of cancer cases has been increasing. In addition because of aging of the population and diversification of values among the people, less-invasive and high-QOL (quality of life) treatment for tumors has been required. BNCT (Boron Neutron Capture Therapy) is known to be a less-invasive tumor therapy based on the reaction between boron $\left({ }^{10} \mathrm{~B}\right)$ and thermal neutron. The reaction cross section is exceptionally large, i.e., $\sim 3800 \mathrm{~b}$ at thermal neutron energy. ${ }^{10} \mathrm{~B}$ thus captures thermal neutron very efficiently and produces energetic $\alpha$ and ${ }^{7} \mathrm{Li}$ particles via ${ }^{10} \mathrm{~B}(\mathrm{n}, \alpha){ }^{7} \mathrm{Li}$ reaction. They have high reactive biological effectiveness (RBE) and the range of them is less than $10 \mu \mathrm{m}$ in tissue ${ }^{1)}$.

However the number of patients treated using conventional BNCT is limited because a nuclear reactor is required as a neutron source of BNCT. In general, nuclear reactors are located far from a hospital and the number of available reactors is few, which are a research reactor. In addition, the patients have to reserve machine time of the nuclear reactors very well in advance, because the main purpose of the nuclear reactors is not a medical use.For these reasons, it is normally difficult for the patients to go to the nuclear reactor with their accompanying doctors, even if they want to select BNCT. Moreover such kinds of atomic reactors do not have enough medical facilities.

Under these circumstances, various studies started so far to realize accelerator-based neutron source for BNCT, because they can be constructed in hospitals in principle. However, such kinds of accelerators are not yet constructed because of various engineering problems. On the other hand, studies on

*Corresponding Author, E-mail:

sakai-m@mb.see.eng.osaka-u.ac.jp development of new agents to deliver ${ }^{10} \mathrm{~B}$ to the tumor are in progress by many scientists, because the BNCT effect is estimated by making the product of ${ }^{10} \mathrm{~B}$ concentration and the thermal neutron flux intensity. If the concentration can be increased, the accelerator load can be decreased substantially.

So we have proposed a new BNCT procedure using accelerator-based neutron source with new boron agents: HVJ (Hemagglutinating Virus of Japan; Sendai virus) envelope (HVJ-E). HVJ-E is a nonviral vector using inactivated virus particle. HVJ-E can deliver a variety of molecules into cells via membrane-fusing activity ${ }^{2}$ and become more than 100 times compared with conventional boron compound (Sodium borocaptate: BSH). Therefore HVJ-E would realize the accelerator-based BNCT.

In this study, we focus on D-T neutron source as an accelerator-based thermal neutron source for BNCT, because if the ${ }^{10} \mathrm{~B}$ concentration could be increased, even a little weak neutron source like the D-T neutron source would be available for BNCT. As for the D-T neutron source, there were some facilities available worldwide. There are two in Japan at present. The staffs in the facilities have enough operation experiences and it would be applicable to BNCT easily. In the past, however, it was confirmed the intensity was a little weak for utilization to BNCT. Now, the situation is changing gradually.

In the present study, to realize accelerator based BNCT as soon as possible, we select combination of D-T neutron source and new boron agent like HVJ-E. The objectives of the study include (1) to construct a thermal neutron field using a D-T neutron source at the Intense $14 \mathrm{MeV}$ Neutron Source Facility, OKTAVIAN, of Osaka University, and (2) to carry out in-vitro irradiation experiments with tumor cells at the thermal neutron field. In the following sections, Monte 
Carlo simulation calculation results for thermal neutron field are discussed by comparing with the experimental results. Also, the anti-tumor effects with the new boron agents by in-vitro experiments are detailed.

\section{Experiments}

\section{D-T Neutron Source and Thermal Neutron Field}

The intense $14 \mathrm{MeV}$ neutron source facility OKTAVIAN of Osaka University was used as a D-T neutron source. OKTAVIAN is a Cockcroft Walton type accelerator and produces $14 \mathrm{MeV}$ neutrons by D-T reaction. Because D-T neutron source technology is already well established, the performance is stable and the source can be utilized to various applications quite easily. Only the problem is to treat tritium as a target.

To construct a thermal neutron field with the D-T neutron source, beryllium and polyethylene blocks were put in between the neutron source and a sample box for irradiation. They were surrounded by a lot of graphite blocks as shown in Fig. 1 to reflect neutrons to enhance neutron economy.

We have investigated the whole arrangement of materials like beryllium, polyethylene and graphite, especially examining the width of polyethylene and the thickness of graphite cover.

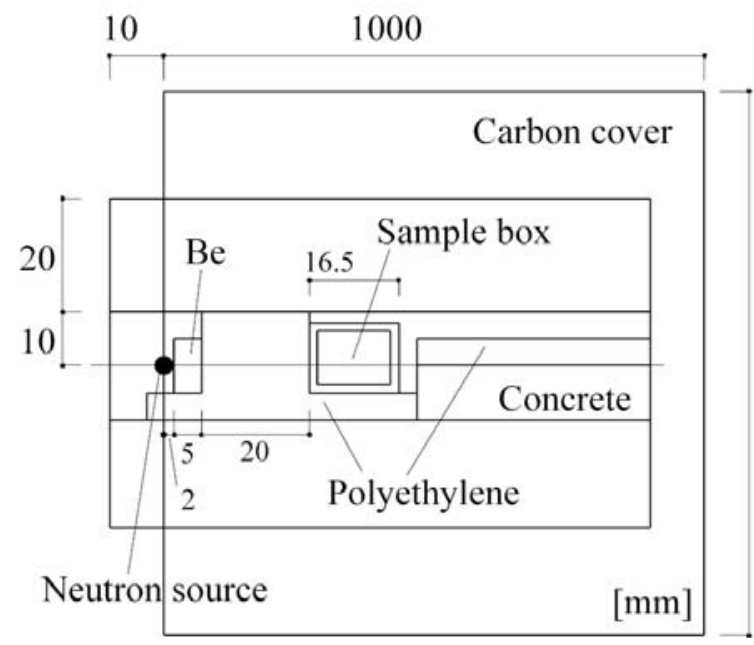

Fig. 1 Experimental set up for neutron irradiation

\section{Monte Carlo Simulation Calculation}

In this study, MCNP-5 (A General Monte Carlo N-Particle Transport Code) was used for neutron transport calculations. The assembly to create a thermal neutron field was exactly modeled according to Fig. 1. The neutron flux was calculated using F4 tally card and reaction rates of various activation foils are estimated with Fm card to compare the experimental results. Absorbed dose for each calculation cell by fast neutrons was calculated using F6 tally card. JENDL-3.2 was used as cross section libarry for neutrons. The number of histories is 5000000 .

\section{Cell Culture}

A549 human lung cancer (adenocarcinoma) cell line was used for these studies. This line was initiated in 1972 by D.J. Giard, et $\mathrm{al}^{3)}$ from lung cancer tissue of a 58 -year-old Caucasian male. A549 cells cultured at $37{ }^{\circ} \mathrm{C}$ and $5 \% \mathrm{CO}_{2}$ in culture medium (RPMI1640 (Sigma-Aldrich Inc., MO)) containing 10\% serum (fetal calf serum, BioWest Inc., France), and antibacterial agent (100 units/mL penicillin, and 0.1 $\mathrm{mg} / \mathrm{mL}$ streptomycin (Nacalai Tesque, Japan)).

\section{Evaluation of Anti-tumor Effect of BNCT}

$100 \mu \mathrm{L}$ of each of $100 \mathrm{ppm}$ B boron agents (BSH, HVJ-E-BSH) was added to $100 \mu \mathrm{L}$ of A549 cell suspension $\left(1 \times 10^{5}\right.$ cells $\left./ \mathrm{mL}\right)$. After 2 hours incubation, the cells were subjected to neutron irradiation for 2 hours. After neutron irradiation, the boron agents were replaced with complete growth medium and cultured 6-well plate (microplate 6-well with Lid. code 3810-006, IWAKI, Japan). After 5 days, the number of cells was counted to evaluate their sensitivity to BNCT. Trypan blue staining methods was used to determine cell viability in this experiment.

\section{Measurement of Fast and Thermal Neutron at Sample Box and Tritium Target}

D-T and thermal neutron flux intensities were monitored with activation foils. For D-T neutrons niobium foils were used and gold foils were for thermal neutrons. Niobium foils of $100 \mu \mathrm{m}$ thickness were put in front of the tritium target and in the sample box as shown Fig. 1. The areas of the niobium foils for both paces were $1 \times 1 \mathrm{~cm}^{2}$ and $5 \times 5 \mathrm{~cm}^{2}$ respectively. Gold foils of $50 \mu \mathrm{m}$ thickness and $1 \mathrm{x} 1$ $\mathrm{cm}^{2}$ were set up along the beam axis and the line perpendicular to the axis to measure 2-dimensional flux distribution. Also cadmium covers of $1 \mathrm{~mm}$ thickness were used together to determine the so-called cadmium ratio. 934 $\mathrm{keV}$ and $412 \mathrm{keV} \gamma$ rays for niobium and gold foils, respectively, were counted using a high-purity germanium (HpGe) (GMX-15185-p, SEIKO EG \& G, Japan). The absolute efficiency of the detector was measured with standard gamma-ray sources. Also, signals from a propotional counter were recorded in a MCS mode to accurately estimate the amount of activation for activation foils.

\section{Measurement of fast and thermal neutron at sample box and tritium target}

All the data were expressed as the mean \pm the standard derivation. Statistical significance (defined as $P$ values of less than 0.01) was evaluated based on the unpaired Student's $t$ test (two-tailed).

\section{Results \\ 1. Calculation of Neutron Flux in Sample Box.}

The thermal, epithermal and fast neutron fluxes at the sample box were shown in Fig. 2 as a function of polyethylene thickness between the tritium target of OKTAVIAN and sample box. From this figure $\sim 20 \mathrm{~cm}$ in 
width is the most suitable.

The reason is in the following:

When the polyethylene thickness was $10 \mathrm{~cm}$, the thermal neutron flux intensity becomes its maximum. However, for example at $20 \mathrm{~cm}$ the thermal neutron flux intensity is not so decreasing but the fast neutron component is rapidly decreasing with increase of thickness. We thus adopted a 20 $\mathrm{cm}$ thick polyethylene between the target and the sample box. Also, as it was pointed out from the past that the thermal neutron flux intensity is ideally obtained to be more-or-less 4 orders of magnitude smaller than the source intensity. In Fig. 2, a similar result is confirmed. Figure 3. shows the neutron spectrum at the irradiation filed.

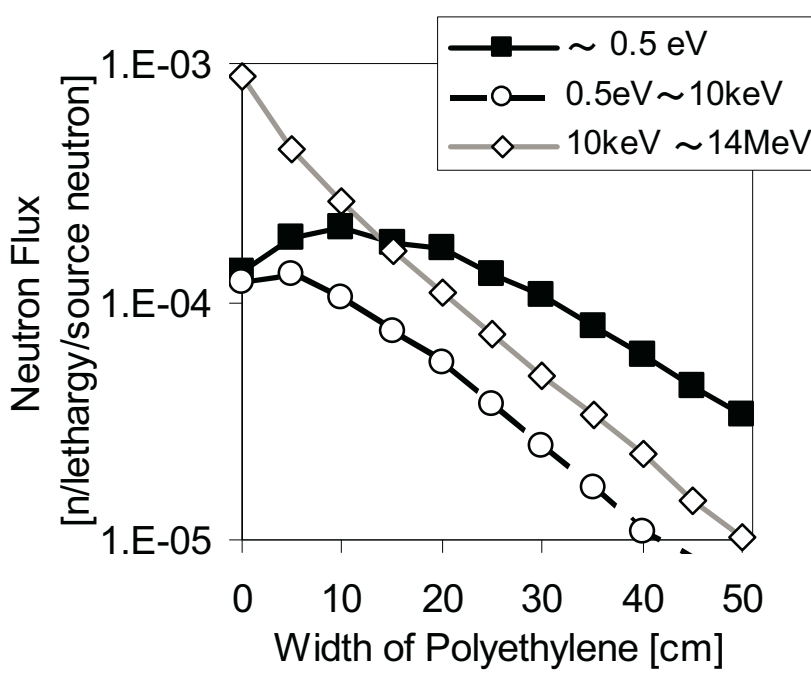

Fig. 2 Neutron flux vs. each width of polyethylene

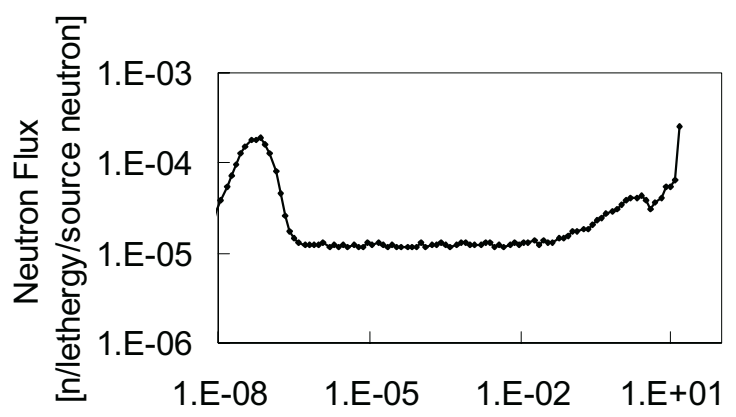

Energy [MeV]

Fig. 3 Neutron spectrum at irradiation field

\section{Measurement of Fast and Thermal Neutron at the Sample Box}

The neutron output from OKTAVIAN was $2.3 \times 10^{10} \mathrm{n} / \mathrm{sec}$. The fast neutron flux was $1.7 \times 10^{6} \mathrm{n} / \mathrm{cm}^{2} / \mathrm{sec}$. The thermal neutron flux was $1.6 \times 10^{6} \mathrm{n} / \mathrm{cm}^{2} / \mathrm{sec}$ and the $\mathrm{Cd}$ ratio was 5.6 . The absolute values are not large, however, irradiation

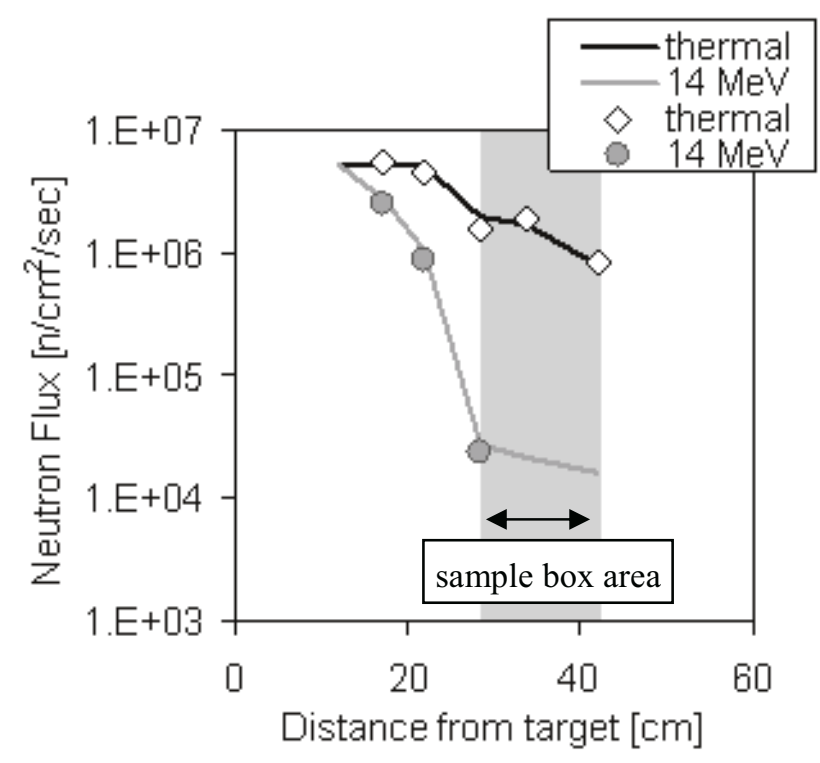

Fig. 4 Neutron flux in set-up (the experimental measurements were compared with simulation result. black line: simulation of thermal, gray line: simulation of fast neutron, white square: experiment of thermal and gray circle: experiment date of fast neutron.)

experiments with the new boron agents are expected to be possible. We compared experimental measurement with MCNP calculation results as shown in Fig. . The simulation values were normalized to the data of gold foil positioned at $10 \mathrm{~cm}$ in depth. The agreement is excellent, i.e., the tendency of the curve for thermal and fast neutrons is almost the same for measurement and calculation. As shown in Fig. , it can also be confirmed the inside the sample box the thermal neutron flux intensity is not fluctuating largely. This is an important point $t$ perfume basic irradiation experiments for BNCT.

\section{Evaluation of the antitumor activity of BNCT}

The cytocidal effects of BNCT were evaluated for each boron agent. The results are described in Fig. . The figure shows the proportion of cell number compared with untreated cell number. Only when using HVJ-E-BSH, the cytotoxicity of BNCT was observed with significant differences. 


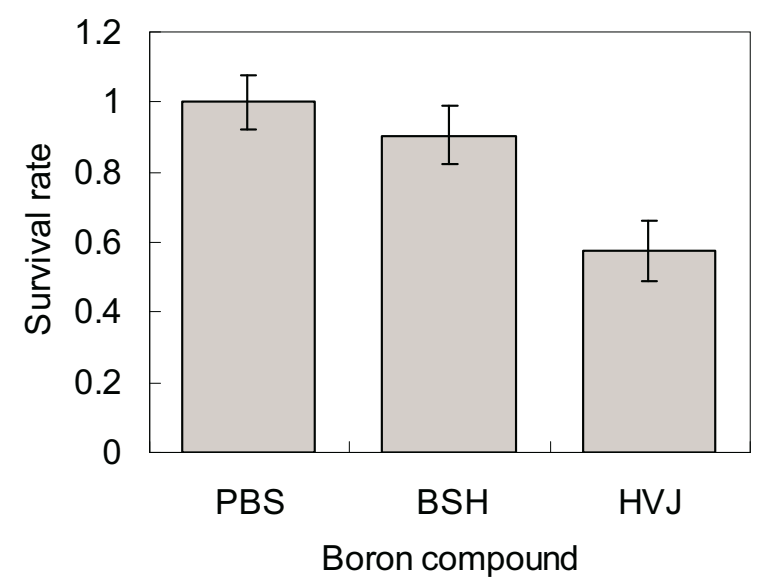

Fig. 5 Effects of BNCT on the Survival.

\section{Discussion}

From the result shown in Fig. , in case of using HVJ-E-BSH, the cytotoxicity of BNCT was observed. It is expected increasing the intracellular boron concentration made the anti-tumor effect. The equivalent dose of BNCT can be calculated by following equations.

$\mathrm{D}_{\mathrm{BNCT}}=\mathrm{RBE} \times \mathrm{K} \times C_{B} \times \phi_{\text {thermal }}$

where; $\mathrm{D}_{\mathrm{BNCT}}$ was the equivalent dose of BNCT. RBE, K, $C_{B}$ and $\phi_{\text {thermal }}$ were Relative Biological Effectivness, kerma factor, boron concentration, and the flux of thermal neutron. The kerma factor was $7.6\left[\mathrm{~Gy} / \mathrm{n}_{\mathrm{th}} / \mathrm{ppm}\right]$ and $\mathrm{RBE}$ of $3.2^{4)}$ was adopted.

In the present case, the equivalent dose was 3.4 Gy-eq, and it was sufficient to observe the cytocidal effects ${ }^{5), 6)}$. On the other hand, since the fast neutron flux intensity was $1.7 \times 10^{6}$ $\mathrm{n} / \mathrm{cm}^{2} / \mathrm{sec}$, the dose was sufficiently-low to show cytotoxicity.

The equivalent dose in the present case was not enough for an actual therapy. However we know the stably produced maximum neutron intensity is an order of $10^{12} \mathrm{n} / \mathrm{s}$ in a D-T neutron source like OKTAVIAN. Also, in our previous work, the intracellular boron concentration using HVJ-E-BSH was enhanced up to $100 \mathrm{ppm}$. Considering these and the presently obtained cytocidal effects, it would be possible to use a D-T neutron source as a thermal neutron source for BNCT.

\section{Conclusion}

Conventional BNCT is inefficient because atomic reactor is used for neutron source. In this study, we demonstrated accelerator-based BNCT using new boron agents.

In this study, HVJ-E can realize the high cytotoxicity reaction compared with conventional boron compound (BSH). Our current approach has a potential for development of accelerator-based BNCT.

We are planning to observe the boron concentration and thermal neutron flux dependency of anti-tumor effects and put in vivo experiment.

\section{References}

1) Kobayashi T., The Present Status and the Future Prospects of Neutron Capture Therapy : A Viewpoint of Radiation Medical Phys-ics and Engineering, Japanese Journal of Radiological Technology, 56[6], 780 (2000)

2) Mima $H$, et al., Biocompatible polymer enhances the in vitro and in vivo transfection effeiciency of $\mathrm{HVJ}$ envelope vector, The J. Gene Medicine, 7, 888 (2005)

3) DJ. Giard, et al., In vitro cultivation of human tumors: establishment of cell lines derived from a series of solid tumors., J. Natl. Cancer Inst., 51[5], 1417 (1973)

4) Coderre, et al., Derivations of relative biological effectiveness for the high-let radiations produced during boron neutron capture irradiations of the $9 \mathrm{~L}$ rat gliosarcoma in vitro and in vivo, Int. J. Radiat. Oncol. Biol. Phys., 27, 1121 (1993)

5) M. Nitsche, et al., The combined effect of fludarabine monophosphate and radiation as well as gemcitabine and radiation on squamous carcinoma tumor cell lines in vitro, Int. J. of Radiat. Biol., 84[8], 643 (2008)

6) R Bromley, et al., Predicting the clonogenic survival of A549 cells after modulated $x$-ray irradiation using the linear quadratic model, Phys. Med. Biol., 54, 187 (2009) 